\title{
Glaucoma Detection in Retinal Image using Medial Axis Detection and Level Set Method
}

\author{
G.Jayanthi \\ Master of engineering \\ Parisutham Institute of Technology \\ \& Science, Thanjavur, \\ Affiliated to Anna University, \\ Chennai- Tamilnadu India
}

\author{
G.Mary Amirtha Sagayee \\ Associate Professor of ECE \\ Parisutham Institute of Technology \\ \& Science, Thanjavur, \\ Affiliated to Anna University, \\ Chennai- Tamilnadu India
}

\author{
S. Arumugam, Ph.D \\ Principal \\ Nandha Engineering College, \\ Erode, Tamilnadu-India
}

\begin{abstract}
Glaucoma is an eye disorder that characterized by elevated Intraocular pressure (IOP). The optic nerve head was damaged by the increased intraocular pressure. It will lead to vision loss, if it is unnoticed. By the extraction of optic disc and optic cup and also calculating the cup to disc ratio, the glaucoma will be detected. In our project we automatically extracted the optic disc in retinal image by using LDA and Medial axis detection. The optic cup extracted by using threshold based initialization level set method and ellipse fitting algorithm. These methods have been tested on drive databases. The average value obtained for (optic disc is a precision value and Recall value are 0.9 and 0.966 respectively, the F-score of 0.9323 and for optic cup a precision value and Recall value are 0.9 and 0.946 respectively, the F-score of 0.9218) describes that this method is a robust tool for detection of optic disc and optic cup..
\end{abstract}

\section{Keywords}

Optic disc, LDA, Cup, boundary detection, Vessel bend, Level set method, cup-to-disc ratio

\section{INTRODUCTION}

In the $21^{\text {st }}$ century, Glaucoma diagnosis and treatment is one of the changeling tasks. In the increased life expectancy and population growth, the number of people was suffered from vision loss due to glaucoma. [1-4]

Diseases are characterized by damage of optic disc, optic nerve and blood vessel enter the retina, detected of optic cup refers to glaucoma. It can only be prevented and cannot be cured. So when detected early and treated to avoid preventable vision loss in the retinal, it can prevent the blindness due to glaucoma. [5-9]

The detection of IOP is not an accurate measurement predict the glaucoma. So in the clinical practice the CDR is one of the main indicators to detect the diseases called glaucoma. The nerve fibers contain in the OD region where the cup is within the OD without fibers. The level of glaucoma increased when the cup area enlarge due to ganglion cell death. The disc area mostly occupies by the cup. By calculating the cup area and disc area and finding the ratio between these areas. If the CDR exceeds a certain value, the glaucoma may be suspected. [1012].There are various method to detect the OD have been proposed in the past but only the fewer method to detect the CDR. The OD boundary detected by the medial axis detection using vessel bends at the OD. Due to the interweavement of blood vessel with surrounding tissues around the cup area, the segmentation of optic cup is the challenging task. So the segmented cup is normally smoothened and an ellipse fitting algorithm is generated to better estimate of cup boundary. [13 16] [21 -22].

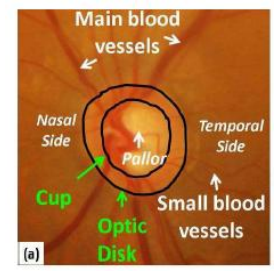

Fig 1: (a) optic disk

OD and cup segmentation is also relevant for automated diagnosis of different ophthalmic pathologies. One amongst them and perhaps the foremost noteworthy is Glaucoma. It is the second most common cause of blindness worldwide. Glaucoma is known by recognizing the amendment in form, color, or depth that it produces within the OD. Thus, its segmentation and analysis can be used to detect of Glaucoma automatically. [17-20][23]

\section{METHODOLOGY}

The objective is to segment the optic disc and optic cup region from the retinal image and finding the CDR. The Fig (2) shows the system architecture. In this approach it has three major modules. They are preprocessing, OD \& Cup segmentation and CDR measurements. Finally, by this CDR measurement the diseases are diagnosed. The first step of preprocessing is applying the LDA to find as much class discriminative information as possible from set of image processing operation. It also converts the colour retinal image for accurate detection of OD. The Segmentation of OD can be done by using medial axis method using vessel bends and boundary is smoothening by $2 \mathrm{D}$ spline interpolation. Next the cup boundary is found by using level set algorithm and boundary is smoothening by ellipse fitting algorithm. These methods are described in below section.

\subsection{Preprocessing}

\subsubsection{Linear Discriminant Analysis}

The goal of LDA is to perform the dimensionality reduction" while conserving as much of the class inequitable information as possible". And try to find direction along which classes best preeminent. Consider the scatter within -classes and between the classes. The work flow of LDA is shown in the Fig (3).

Step1: Original image is into ycbcr and represent the image as matrix. 
Step2: Compute the mean of each data $(\mu 1$ and $\mu 2)$ and mean of entire data $\mu 3$

$$
\mu 3=\mathrm{p} 1 * \mu 1+\mathrm{p} 2 * \mu 2
$$

Step3: Between -class scatter and with in class scatter matrix is computed by using $\mathrm{Sb}=\sum(\mu \mathrm{j}-\mu 3)^{*}(\mu \mathrm{j}-\mu 3)^{-\mathrm{T}} \quad$ \& $\mathrm{Sw}=\sum(\mathrm{xj}-\mu 1 \mathrm{j}) *(\mathrm{xj}-\mu \mathrm{j})^{\mathrm{T}}$

Step4: Finding the eigen value by using $\lambda=\mathrm{Sw}^{-1} \mathrm{Sb}$.

Step5: If eigen values are non-zero, then it is linearly independent between feature is indicated. If eigen values are zero, then it is linearly dependent between features is indicated and its eigen values are neglected.

The input image to LDA is color retinal fundus image as shown in the Fig. 4(a). The color image is converting into ycbcr matrix form for finding the eigen values and finally it converted into grey scale image to differentiate the structure of retina more clearly as shown in the Fig 4(b).

\subsubsection{Image enhancement}

The local transformation is used to correct the non-uniform illumination and improve its contrast. Its expression is given in the equation 1.

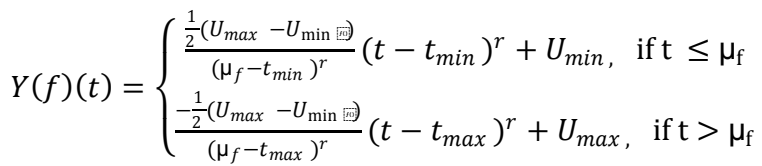

The minimum and maximum grey level of image is $t_{\min }$ and $t_{\max }$. The mean value of the image is $u_{f}$ for all the pixels within a window centered at the current pixel $x$. The increasing contrast is control by the parameter $r$. The output of image enhancement by the nonlinear local transformation $\mathrm{Y}$ is shown in the Fig 4(c)

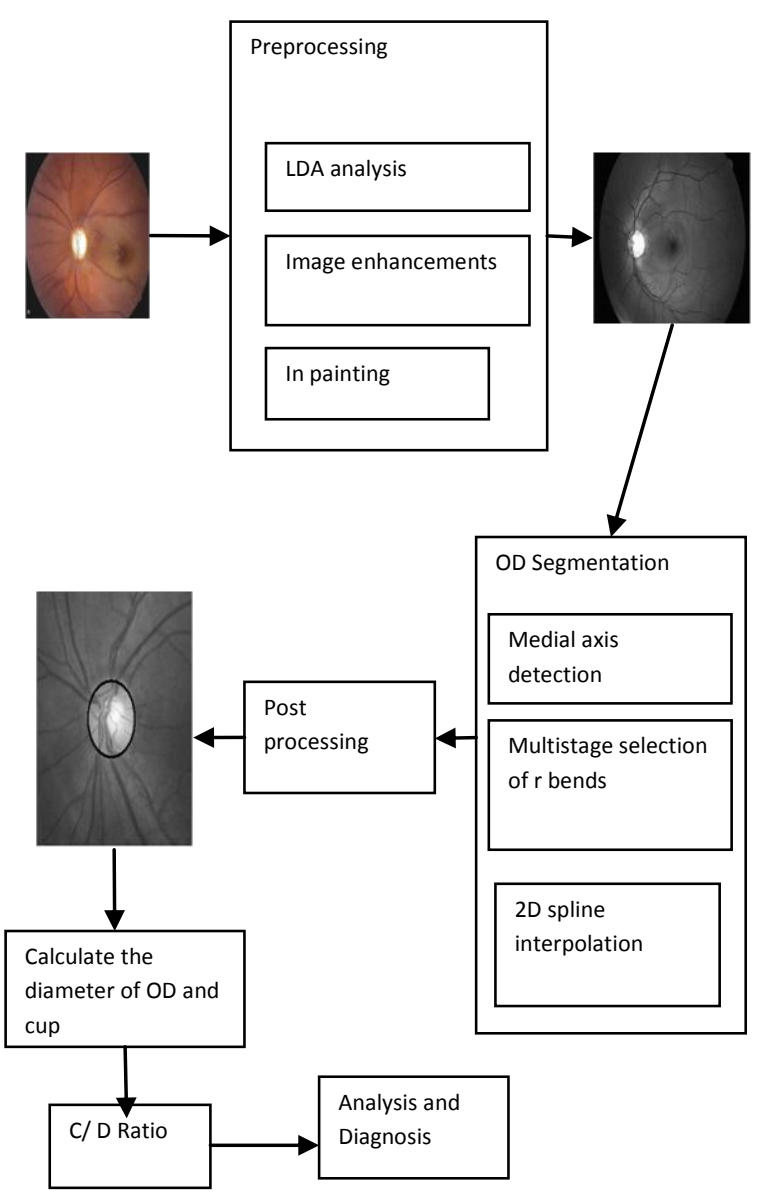

Fig 2: The proposed system architecture

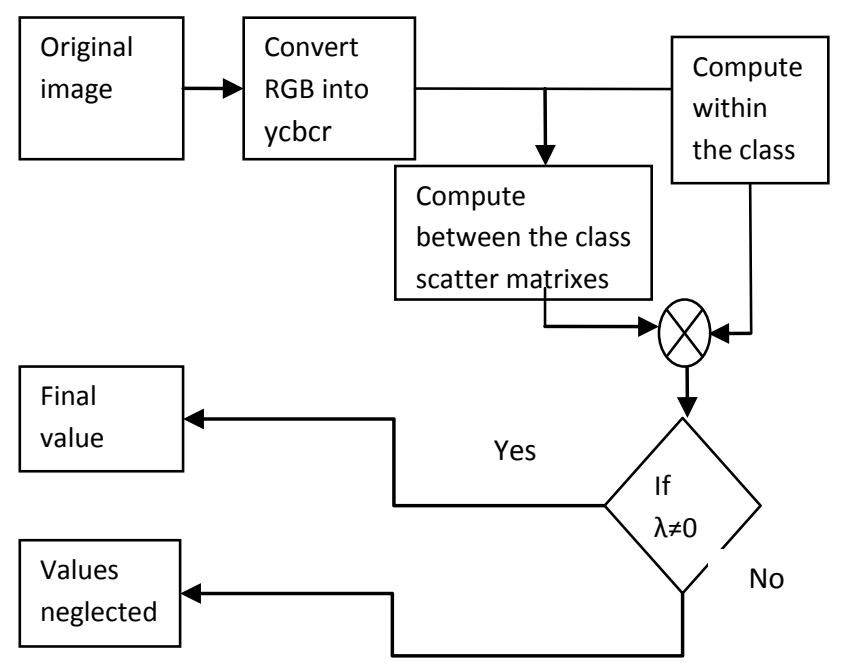

Fig 3: Flow chart for LDA 


\subsubsection{Vessel mask}

There are totally 0 to 255 pixels. Initially it assign zero for black and white. Calculate the rows and columns pixel value. If the range is between 0 to150 then it represents as black. If it is above 220 , it represents as white and range between 150 and 220 represents as grey level. Set the threshold value as $0.10 \%$. The pixels within the threshold consider as white and out of the threshold consider as black. The Fig 3(d) and 3(e) shows the vessel masking and inpainted image.

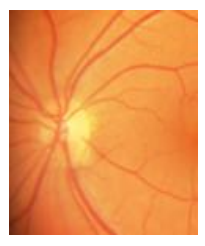

(a)

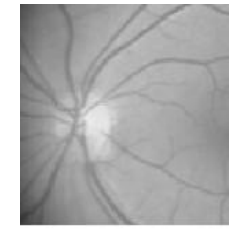

(b)

(c)
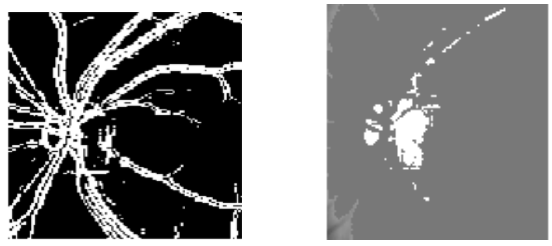

(d)

(e)

Fig 4: a) Original fundus image. (b) LDA. (c) Image enhancement (d) Vessel Masking (e) Inpainted

\subsection{Optic disc Segmentation}

A multiple source of information is needed to segment the optic disc. The pallor region describes the inner limit of $r$ bends, bending angle and the location of OD. The $r$ bends are not equally distributes in the OD boundary. 2D spline interpolations are used to smooth the OD boundary.

\subsubsection{Medial axis detection}

Due to large inter-image variation, detecting both thick and thin vessels are difficult. The curvature is computed by using surface tangent derivatives and it is given in the equation (2).

$$
\mathrm{Y}(\mathrm{x})=\left(\mathrm{d} 2 \mathrm{y} / \mathrm{dx}^{2}\right) /\left(1+(\mathrm{dy} / \mathrm{dx})^{2}\right)
$$

$\mathrm{Y}(\mathrm{x})$ is computed in 4 different directions for each point. To obtain the trench point, maximum value of $\Upsilon \max$ and its corresponding orientation $\alpha$ are used. If the value of Ymax is greater than both the threshold value $t$ and neighboring pixel in $\alpha$ direction, then it is declared as trench point. A high contrast of vessel point (set 1 ) is obtained by applying the high value of t. Similarly a low contrast vessel point (set 2) is obtained by applying the value of $t$. This helps to extract the low contrast vessel while rejecting the noise. The final trench point detect the vessel structure more accurately quantify the vessel bends through medial axis detection. The Fig 5(a) shows Medial axis detection of the OD.

\subsubsection{Vessels bends detection}

The vessel bends are varied according to the caliber vessel. Due to inflexibility of thick vessel it doesn't show large bending but thin vessel shows large bending. First, the vessel segments are extracted by terminated end points. The local maxima are compute by $1 \mathrm{D}$ shape profile and constitute a set of bends. A segment of vessel around bi and bound on either side by nearest minimum curvature defines the region of support. The line joining a bend point and centre of the mass of ROS has been used to compute the bending angle $\theta$. The bending angle which exceeds $170^{\circ}$ then it has to be removed from the set of bends. Fitted area and bend points are shown in the Fig 5(b) and 5(c).

\subsubsection{Multi-state selection of $r$ bends}

The task of identifying the r-bends from bi is performed in two stages to reduce the required analysis, by utilizing anatomical knowledge associated with r-bends. In the first stage, a coarse selection is done based on a bend's proximity to the pallor region. In the second stage, the spatial position and bending information are used to identify the set of $r$ bends.

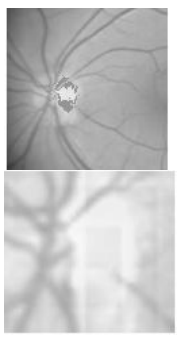

(a)
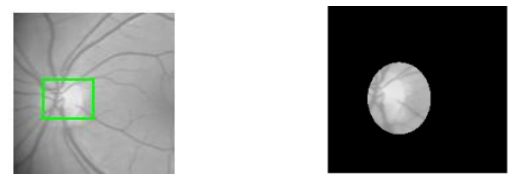

(b)

(c) (d)
Fig 5: OD Segmentation (a) Medial axis detection (b) Fitted area (c) bend points (d) optic vessel

A sector is radially analyzed with step size of $20^{\circ}$ and in each step, only bends formed by vessel with correct orientation are retained. The optic vessels are shown in the Fig 5(d).

\subsubsection{D spline interpolation}

The goal of spline interpolation is to get an interpolation formula that is continuous in both the first and second derivatives, both within the intervals and at the interpolating nodes. This will give us a smoother interpolating function.

\subsection{Optic Cup Segmentation}

The Segmentation of optic cup boundary is more defying than disc segmentation. This is due to interweavement with the cup and the rim is often not as important as that of the disc boundary. In this paper we proposed the threshold -initialized level set function. In this the histogram analysis is used threshold value is used to determine the region of interest. To define the initial contour in the ROI, top $1 / 3$ of the grayscale intensity was used. The cup segmentation method uses level set method to increase the accuracy. The functional block diagram of cup segmentation is shown in the Fig (6). 


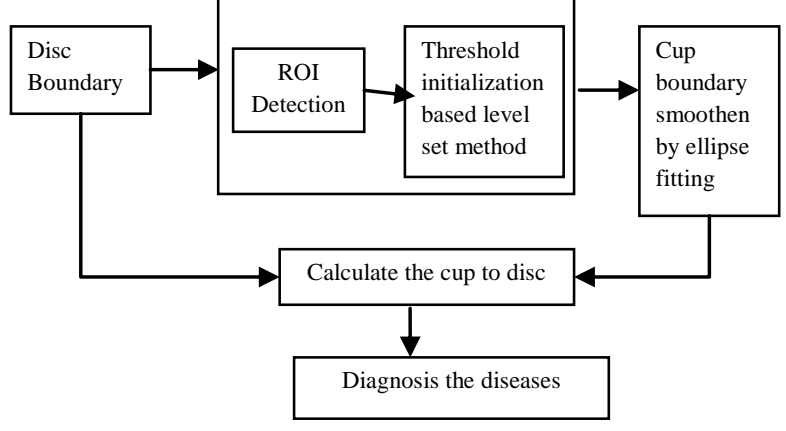

Fig 6: Functional block of cup segmentation

\subsubsection{Threshold initialization based level set method}

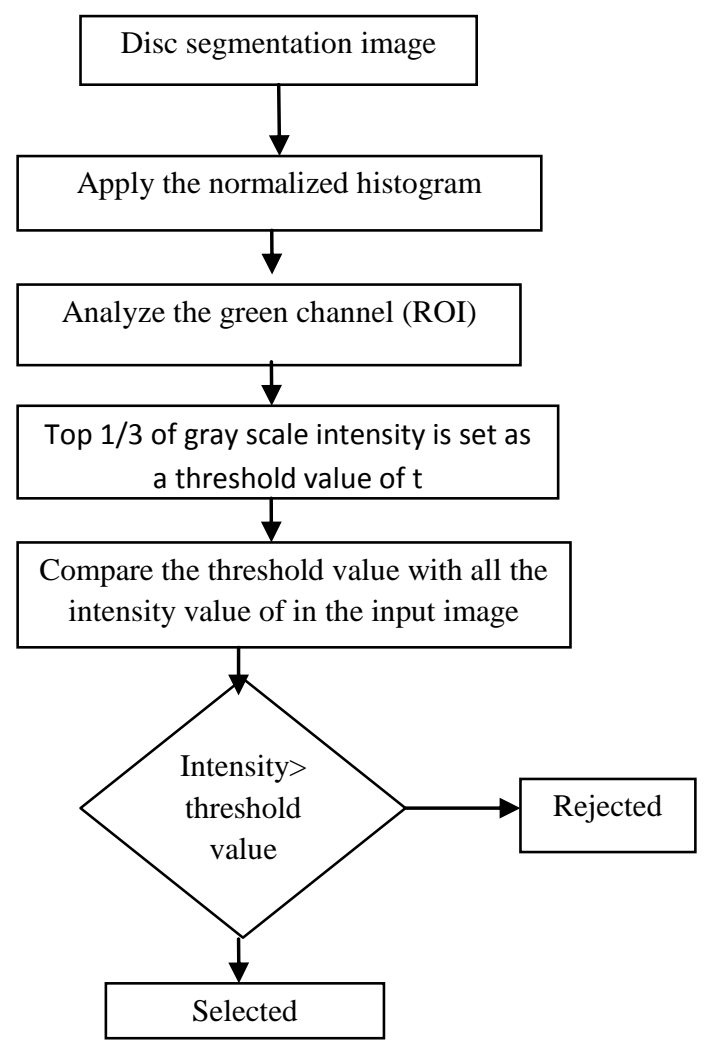

Fig 7: Flow diagram of level set algorithm

The Fig 7 shows the working flow diagram of threshold-initialization level set method.

Step 1: The green channel of the input image is selected as the basis for further segmentation due to the optimum observed contrast between the cup and disc boundaries in this channel.

Step 2: Then the normalized cumulative histogram of the green channel image is analyzed and a threshold value which segments out the pixels.

Step3: The top $1 / 3$ of the grayscale intensity is used to define the initial cup contour.

Step4: The top $1 / 3$ of the grayscale intensity is to find the threshold value from the normalized cumulative histogram.
Step5: compare the threshold value with all the intensity values of the input image.

Step 6: The intensity value is that is greater than the threshold value is selected. The Fig 8(a) and 8(b) shows the histogram analysis of the image and fitted image.

\subsubsection{Algorithm for optic cup extraction}

Step1: Morphological operation such as dilation and erosion are applied on the selected image to preserve the essential shape and eliminate the noise.

Step2: Apply the morphological gradient operation that highlights gray level transition in the input image.

Step3: Apply the median filtering for image blurring and remove the impulse noise.

Step4: Apply the Least square ellipse fitting algorithm to smoothen the boundary.

Step 5: Optic disc area $=$ No. of pixel in that disc

Optic cup area $=$ No. of pixel in that area.

Step6:

$$
C D R=\frac{\text { No.of pixel in the optic cup area }}{\text { No.of pixel in the optic disc area }}
$$

\subsubsection{Morphological operation}

\section{Dilation}

In the mathematical morphology the dilation is one of the basic operations. First it expands the image to grayscale and to lattices. By using a vector addition of set element, dilation combines the two sets. It makes the output image are brighter than input image and expand it. The Fig 8 (d) shows the dilation of the image.

\section{Erosion}

From the set of the element the vector subtraction is used to combine the two sets and it shrinks the object.
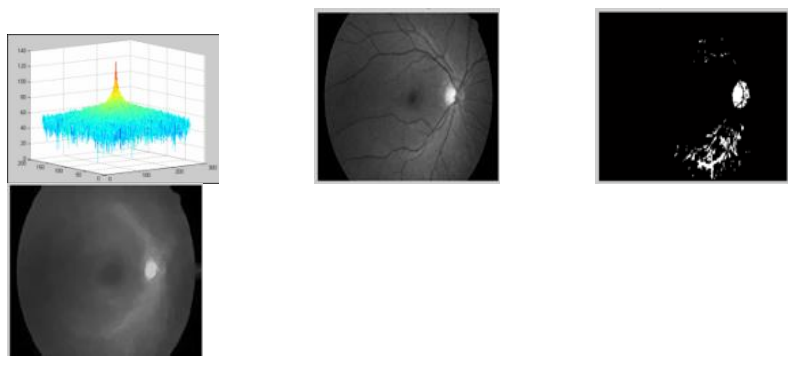

(a)

(b)

(c) 


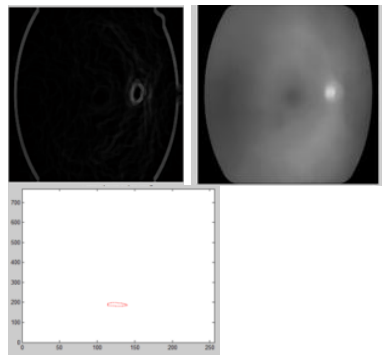

(e) (f)

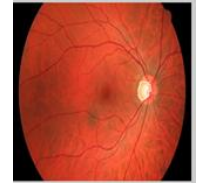

(g)

(h)
Fig 8: Cup segmentation. (a) Histogram image (b) Filtered image (c) segmented OD (d) Erosion (e) Morphological Gradient (f) Median filtered (g) Segmented cup (h) Ellipse Feature point.

\section{Morphological Gradient:}

The difference between the dilation and erosion of a given image is known as Morphological gradient. The gray level transition in the image is highlighted by Morphological gradient. The optic cup boundary is easily identified by it. The Morphological gradient image is shown in the image 8(e)

\subsubsection{Ellipse Fitting algorithm}

To smooth the cup boundary the ellipse fitting algorithm is used. It is usually based on least square fitting algorithm. Assume the minimal sum of the deviation from given data points gives the best fit curve. The B2AC method further constrains the parameter vector a in such a way that it forces the conic to be an ellipse through imposing the equality constraint. The segmented cup and ellipse feature point is shown in the Fig 7(g) and 7(h).

\section{PERFORMANCE EVALUATION OF DISC AND CUP BOUNDARY}

The overall performance of the OD boundary was passed by the comparative analysis method. The boundary detection similarity is done by the evaluation process. The area overlap between computed region and ground was evaluated by precision and recall values. The expression for Precision and Recall values are given in the equation 3 and 4 .

$$
\text { Precision }=\frac{T p}{T p+F p}
$$

$$
\text { Recall }=\frac{T p}{T p+F n}
$$

$\mathrm{Tp} \rightarrow$ number of true positive pixels. $\mathrm{Fp} \rightarrow$ number of false positive pixels, Fn $\rightarrow$ number of false negative pixels. To compute a single performance measure called traditional Fscore $(\mathrm{F})$ is calculated by the equation 5 .

$$
F=\frac{2 \text { Precision } * \text { Recall }}{\text { Precision }+ \text { Recall }}
$$

The Value of F-score should lie between 0-1 and for accurate method the F-score should be high. The Performance of disc boundary of various techniques has been evaluated with small data base. From the various techniques, the proposed method detect the disc boundary more accurately with the precision value of 0.9 , recall value of 0.966 and F-Score of 0.9323 . The Performance of Cup boundary are detected more accurately with precision value of 0.9 , recall value of 0.94732 and the F-score value of 0.9218 and also calculating the cup to disc ratio to detect the glaucoma diseases. The normal range of CDR is in the range of 0.1 to 0.3 . If it is exceeds this range it indicated the glaucoma disease in the retinal image.

TABLE-I

PERFormance EVALUATION OF DISC BOUNDARY BY VARIOUS TECHNIQUES

\begin{tabular}{|l|c|c|c|}
\hline \multicolumn{1}{|c|}{ TECHNICQUES } & PRECISION & RECALL & F-SCORE \\
\hline $\begin{array}{l}\text { Hausdorff-based } \\
\text { template }\end{array}$ & 0.94 & 0.716 & 0.81 \\
\hline $\begin{array}{l}\text { Morphological } \\
\text { method }\end{array}$ & 0.773 & 0.836 & 0.80 \\
\hline Supervised method & 0.98 & 0.70 & 0.82 \\
\hline SVM & 0.85 & 0.84 & 0.84 \\
\hline Hough Transform & 0.99 & 0.82 & 0.90 \\
\hline Fuzzy Convergence & 0.99 & 0.85 & 0.91 \\
\hline $\begin{array}{l}\text { Active contour } \\
\text { Method }\end{array}$ & 0.72 & 0.82 & 0.81 \\
\hline $\begin{array}{l}\text { Proposed } \\
\text { Method(Medial } \\
\text { Axis Detection) }\end{array}$ & 0.9 & 0.966 & 0.9323 \\
\hline
\end{tabular}


TABLE-II

PERFormance EVALUATION OF CUP BOUNDARY By VARIOUS TECHNIQUES

\begin{tabular}{|l|c|c|c|c|l|}
\hline \multicolumn{1}{|c|}{$\begin{array}{c}\text { Test } \\
\text { Images }\end{array}$} & $\begin{array}{c}\text { Precision } \\
\text { Value }\end{array}$ & $\begin{array}{c}\text { Recall } \\
\text { Value }\end{array}$ & F-Score & CDR & $\begin{array}{c}\text { Diseases } \\
\text { Diagnosis }\end{array}$ \\
\hline $\begin{array}{l}\text { Test } \\
\text { Images 1 }\end{array}$ & 0.9 & 0.95 & 0.924 & 0.086 & Normal \\
\hline $\begin{array}{l}\text { Test } \\
\text { Images 2 }\end{array}$ & 0.9 & 0.9425 & 0.9207 & 0.139 & Normal \\
\hline $\begin{array}{l}\text { Test } \\
\text { Images 3 }\end{array}$ & 0.9 & 0.93 & 0.914 & 0.038 & Normal \\
\hline $\begin{array}{l}\text { Test } \\
\text { Images 4 }\end{array}$ & 0.9 & 0.96 & 0.92903 & 1.301 & Affected \\
\hline $\begin{array}{l}\text { Test } \\
\text { Images 5 }\end{array}$ & 0.9 & 0.93 & 0.91475 & 0.236 & Normal \\
\hline $\begin{array}{l}\text { Test } \\
\text { Images 6 }\end{array}$ & 0.9 & 0.95 & 0.92432 & 0.0410 & Normal \\
\hline $\begin{array}{l}\text { Test } \\
\text { Images 7 }\end{array}$ & 0.9 & 0.93 & 0.91 & 0.350 & Affected \\
\hline $\begin{array}{l}\text { Test } \\
\text { Images 8 }\end{array}$ & 0.9 & 0.9956 & 0.94 & 4.55 & Affected \\
\hline $\begin{array}{l}\text { Test } \\
\text { Images 9 }\end{array}$ & 0.9 & 0.925 & 0.91275 & 0.0174 & Normal \\
\hline $\begin{array}{l}\text { Test } \\
\text { Images 10 }\end{array}$ & 0.9 & 0.96 & 0.92903 & 0.0344 & Normal \\
\hline
\end{tabular}

\section{CONCLUSION}

In this paper, a disk boundary detection using medial axis detection method was proposed. First, the LDA method is used to differentiate more clearly to detect the OD more accurately. Next the trench based vessel modeling and ROS based bend detection is used to detect the variation of the vessel thickness. Finally the 2D spline interpolation is applied on detected $\mathrm{r}$ bends to smoothen the OD boundary. The optic cup extracted by using threshold based initialization level set method and ellipse fitting algorithm. In this method, it detected OD boundary accurately with precision of 0.9 \& recall of 0.966. The F-Score of the detected boundary is 0.9323 and the cup boundary with precision of $0.9 \&$ recall of 0.946 . The F- Score of the detected cup boundary is 0.9218 .

\section{REFERENCES}

[1] Sandra Morales*, Valery Naranjo, Jesús Angulo, and Mariano Alcañiz, Automatic Detection of Optic Disc Based on PCA and Mathematical Morphology, IEEE Trans.Med.Imaging VOL. 32, NO. 4, APRIL 2013.

[2] Adam Hoover, "Locating the Optic Nerve in a Retinal Image Using the Fuzzy Convergence of the Blood Vessels", Michael Goldbaum.

[3] Arturo Aquino, "Detecting the Optic Disc Boundary in Digital Fundus Images Using Morphological, Edge Detection, and Feature Extraction Techniques", Manuel Emilio Gegúndez-Arias, and Diego Marín

[4] T. R. G. Babu and S. Shenbagadevi. Automatic detection of glaucoma using fundus image. European Journal of Scientific Research, 59:22-32, 2011

[5] R. Bock, J. Meier, L. G. Nyl, and G. Michelson, "Glaucoma risk index: automated glaucoma detection from color fundus images," Medical Image Analysis, vol. 14(3), pp. 471-481, 2010.

[6] Diego Marín, "A New Supervised Method for Blood Vessel Segmentation in Retinal Images by Using GrayLevel and Moment Invariants-Based Features", Arturo Aquino*, Manuel Emilio Gegúndez-Arias, and José Manuel Bravo

[7] Y. Fengshou. Extraction of features from fundus images for glaucoma assessment. Master's thesis, National University of Singapore, 2011.

[8] Gopal Datt Joshi, Member, IEEE, Jayanthi Sivaswamy, Member, IEEE, and S. R. Krishnadas, Optic Disk and Cup Segmentation from Monocular Colour Retinal Images for Glaucoma Assessment.

[9] J. Hajer, H. Kamel, and E. Noureddine, "Localization of the optic disk in retinal image using the water snake," in Proc. Int. Conf. Comput. And Commun. Eng., 2008, pp. 947-951.

[10] Y. Hatanaka, A. Noudo, C. Muramatsu, A. Sawada, T. Hara, T. Yamamoto, and H. Fujita, "Automatic measurement of vertical cup-to-disc ratio on retinal fundus images," Proc. ICMB, pp. 64-72, 2010.

[11] G. D. Joshi, J. Sivaswamy, K. Karan, P. R., and R. Krishnadas, "Vessel bend-based cup segmentation in retinal images," Proc. Int. Conf. Pattern Recognition (ICPR), pp. 2536-2539, 2010.

[12] Juan Xua, "Optic disk feature extraction via modified deformable model technique for glaucoma analysis", Opas Chutatapeb, Eric Sungc, Ce Zhengd, Paul Chew Tec Kuand.

[13] Keith A. Goatman," Detection of New Vessels on the Optic Disc Using Retinal Photographs", Alan D. Fleming, Sam Philip, Graeme J. Williams, John A. Olson, and Peter F. Sharp.

[14] S. Lu, "Accurate and efficient optic disc detection and segmentation by a circular transformation," IEEE Trans. Med. Imag., vol. 30, no. 12, pp. 2126-2133, Dec. 2011.

[15] J. Liu, D. Wong, J. Lim, H. Li, N. Tan, and T. Wong, "Argali- an automatic cup-to-disc ratio measurement system for glaucoma detection and analysis framework," Proc. SPIE, Medical Imaging, pp. 72 603K-8, 2009.

[16] Marc Lalonde, "Fast and Robust Optic Disc Detection Using Pyramidal Decomposition and Hausdorff-Based Template Matching", Mario Beaulieu, and Langis Gagnon*

[17] D. Pascolini and S. P.Mariotti, "Global estimates of visual impairment: 2010,” Br. J. Ophthalmol., pp. 614621,2011

[18] R. J. Qureshi, L. Kovacs, B. Harangi, B. Nagy, T. Peto, and A. Hajdu, "Combining algorithms for automatic detection of optic disc and macula in fundus images," Comput. Vis. Image Understand., vol. 116, no. 1, pp. 138-145, 2012

[19] S. Sekhar, "Automated Localisation of Retinal Optic Disk Using Hough Transform", W. Al-Nuaimy and A. K. Nandi

[20] K. Stapor, A. Sacutewitonski, R. Chrastek, and G. Michelson, "Segmentation of fundus eye images using 
methods of mathematical morphology for glaucoma diagnosis," Proc. ICCS, pp. 41-48, 2004.

[21] Siddalingaswamy P. C. Automatic Localization and Boundary Detection of Optic Disc Using Implicit Active Contours, 2010 International Journal of Computer Applications (0975 - 8887) Volume 1 - No. 7

[22] Toru Tamaki, Bingzhi Yuan, Kengo Harada, Bisser Raytchev, Kazufumi Kaneda Hiroshima University,
Japan Linear Discriminative Image Processing Operator Analysis.

[23] D. Welfer, J. Scharcanski, C. M. Kitamura, M. M. D. Pizzol, L. W. Ludwig, and D. R. Marinho, "Segmentation of the optic disk in color eye fundus images using an adaptive morphological approach,"Comput. Biol. Med., vol. 40, no. 2, pp. 124-137, 2010. 